\title{
Influência do Tratamento Quimioterápico no Comportamento Alimentar e Qualidade de Vida de Pacientes Oncológicos
}

doi: https://doi.org/10.32635/2176-9745.RBC.2019v65n2.93

\author{
Influence of Chemotherapy Treatment on Eating Behavior and Quality of Life of Oncologic Patients \\ Influencia del Tratamiento de Quimioterapia en el Comportamento Alimentario y Calidad de Vida de los Pacientes con \\ Cáncer
}

Ana Letícia Pereira Andrade'; Emilene Maciel e Maciel²; Gilmara Péres Rodrigues ${ }^{3}$; Suelem Torres de Freitas ${ }^{4}$; Maria da Cruz Moura e Silva ${ }^{5}$

Resumo

Introduçáo: $\mathrm{O}$ câncer é uma doença de origem multifatorial, com crescimento irregular e descontrolado de células, cujo tratamento pode desencadear aversóes alimentares e mudanças na qualidade de vida. Objetivo: Avaliar a influência do tratamento quimioterápico no comportamento alimentar e na qualidade vida de pacientes oncológicos. Método: Estudo longitudinal e observacional, realizado de junho a outubro de 2018, no Hospital Universitário da Universidade Federal do Piauí. Os dados foram obtidos em dois momentos: T0, socioeconômicos, demográficos, clínicos, de comportamento alimentar e qualidade de vida; e T1, de comportamento alimentar e qualidade de vida. Foram utilizados os testes Shapiro-Wilk, t-Student e Wilcoxon; Pearson e Spearman com p< 0,05. Resultados: Dos 17 pacientes, a maioria era do sexo feminino $(82,4 \%)$, média de 54,2 anos, renda familiar de um a dois salários mínimos $(64,7 \%)$ e de etnia parda (76,5\%). O câncer mais frequente foi o de mama (52,9\%). Houve aversôes alimentares em T1 para: "sopas e massas" (p=0,001), "carnes e peixes" ( $\mathrm{p}=0,016)$, e "doces, sobremesas e aperitivos" ( $\mathrm{p}=0,001)$. Houve diferença significativa na qualidade de vida quanto à medida global de saúde $(\mathrm{p}=0,001)$ e dificuldade financeira $(\mathrm{p}=0,026)$, assim também como nas correlaçóes entre qualidade de vida e comportamento alimentar. Conclusáo: Os resultados reforçam a necessidade de constante monitoramento nutricional desde o início do tratamento quimioterápico com o intuito de evitar e/ou reduzir suas repercussóes negativas no estado nutricional e, consequentemente, na qualidade de vida. Além disso, a realização de mais estudos, com amostra e intervalo de tempo maiores, é necessária.

Palavras-chave: Neoplasias/tratamento farmacológico; Comportamento Alimentar/efeitos dos fármacos; Qualidade de Vida.

\begin{abstract}
Introduction: Cancer is a multifactorial disease with uncontrolled cells growth, whose treatment can trigger food aversions and changes in quality of life. Objective: To evaluate the influence of chemotherapy treatment on eating behavior and quality of life of oncologic patients. Method: A longitudinal and observational study, carried out from June to October of 2018, at the University Hospital of the Federal University of Piauí. The data were obtained in two moments: T0, socioeconomic, demographic, clinical, food behavior and quality of life, and T1, food behavior and quality of life. The following tests were used: Shapiro-Wilk, t-Student and Wilcoxon; Pearson and Spearman, with $\mathrm{p}<0.05$. Results: Of the 17 patients, the majority were females (82.4\%), medium age of 54.2 years, family income of 1 to 2 minimum wages (64.7\%) and ethnics, brown (76.5\%). Breast cancer was the most frequent $(52.9 \%)$. There were food aversions in T1, for "soups and pastas" ( $\mathrm{p}=0.001)$, "meats and fish" $(\mathrm{p}=0.016)$, and "sweets, desserts and appetizers $(\mathrm{p}=0.001)$. There was a significant difference in quality of life regarding the general health status $(\mathrm{p}=0.001)$ and financial difficulty $(\mathrm{p}=0.026)$, as well as in correlations between quality of life and food behavior. Conclusion: The results reinforce the need of constant nutritional monitoring since the beginning of the chemotherapy in order to avoid and/or reduce the negative repercussions on the nutritional status and, consequently, on the quality of life. In addition, more studies with longer time ranges and larger samples are required.

Key words: Neoplasms/drug therapy; Feeding Behavior/drug effects; Quality of Life.
\end{abstract}

Resumen

Introducción: El cáncer es una enfermedad de origen multifactorial, con crecimiento descontrolado de células, cuyo tratamiento puede desencadenar aversiones alimenticias y cambios en la calidad de vida. Objetivo: Evaluar la influencia del tratamiento quimioterápico en el comportamiento alimentario y en la calidad de vida de pacientes oncológicos. Método: Estudio longitudinal y observacional, realizado de junio a octubre de 2018, en el Hospital Universitario de la Universidad Federal de Piauí. Los datos fueron obtenidos en dos momentos: T0, socioeconómicos, demográficos, clínicos, de comportamiento alimentario y calidad de vida y T1, de comportamiento alimentario y calidad de vida. Se utilizaron las pruebas: Shapiro-Wilk, t-Student y Wilcoxon; Pearson y Spearman, con $\mathrm{p}<0,05$. Resultados: De los 17 pacientes, la mayoría eran mujeres $(82,4 \%)$, promedio de 54,2 ańos, ingreso familiar de 1 a 2 salarios mínimos $(64,7 \%)$ y de etnia parda $(76,5 \%)$. El cáncer más frecuente fue el de mama (52,9\%). Se observaron aversiones alimentarias en T1, para algunos grupos de alimentos. Se observó una diferencia significativa em la calidad de vida en cuanto a la medida global de salud $(\mathrm{p}=0,001)$ y dificultad financiera $(\mathrm{p}=0,026)$, así como las correlaciones entre calidad de vida y comportamiento alimentario. Conclusión: Los resultados refuerzan la necesidad de constante monitoreo nutricional desde el inicio del tratamiento quimioterápico con el fin de evitar y/o reducir lâs repercusiones negativas de este en el estado nutricional y en calidad de vida. Además, la realización de más estúdios com muestra e intervalo de tempo mayor, son necesarios.

Palabras clave: Neoplasias/tratamiento farmacológico; Conducta Alimentaria/efectos de los fármacos; Calidad de Vida.

\footnotetext{
${ }^{1}$ Hospital Universitário da Universidade Federal do Piauí (HU/UFPI). Teresina (PI), Brasil. Orcid iD: https://orcid.org/0000-0002-7721-2090

${ }^{2}$ HU/UFPI. Teresina (PI), Brasil. Orcid iD: https://orcid.org/0000-0002-5743-6687

${ }^{3}$ HU/UFPI. Teresina (PI), Brasil. E-mail: gilmaraperes@ufpi.edu.br. Orcid iD: https://orcid.org/0000-0003-2770-8191

${ }^{4}$ Universidade Federal do Pará. Belém (PA), Brasil. Orcid iD: https://orcid.org/0000-0002-2146-2840

${ }^{5} \mathrm{HU} /$ UFPI. Teresina (PI), Brasil. Orcid iD: https://orcid.org/0000-0002-9181-7838

Endereço para correspondência: Ana Letícia Pereira Andrade. Rua Chanceler Edson Queiroz, 2043 - Dirceu 1. Teresina (PI), Brasil. CEP 64077-750.

E-mail: ana13_leticia@hotmail.com
} 


\section{INTRODUÇÃO}

O câncer é uma doença crônica de origem multifatorial, na qual há crescimento irregular e descontrolado de células que são capazes de se multiplicarem e infiltrarem diversos órgãos e tecidos. Atualmente, é apontado como um problema de saúde pública tanto em países desenvolvidos quanto nos subdesenvolvidos ${ }^{1}$.

Grande parte dos pacientes diagnosticados com câncer consegue receber o tratamento adequado, que cursa com múltiplos efeitos colaterais como: fadiga, dor, dispneia, náuseas, anorexia e perda de peso não intencional. Esses sintomas podem exercer impacto negativo no tratamento, como também na qualidade de vida (QV) desses indivíduos ${ }^{2}$.

$\mathrm{O}$ tratamento quimioterápico pode afetar a dieta e os hábitos alimentares do indivíduo com câncer, por acarretar alteraçôes quimiossensoriais, distorçôes do gosto e aumento na sensibilidade olfativa, em virtude da própria doença ou pelo efeito colateral do tratamento proposto. Tais mudanças repercutem na perda de peso, baixa ingestão alimentar e redução na $\mathrm{QV}^{3}$.

Segundo Palazzo ${ }^{4}$, as aversôes alimentares adquiridas pelos pacientes oncológicos resultam do mal-estar vinculado ao uso de quimioterápicos que afetam negativamente o sabor dos alimentos. Pois, durante o período em que o antineoplásico permanece ativo, as células sensoriais do paladar sofrem alteraçóes que levam à redução da sensibilidade e, consequentemente, dos sabores, contribuindo com a ingestão ou não de alguns alimentos, repercutindo no comportamento alimentar e QV desses pacientes.

De acordo com a World Health Organization (WHO) $)^{5}$, a QV é definida como "a percepçáo do indivíduo de sua posição na vida, no contexto da cultura e sistema de valores nos quais ele vive e em relação aos seus objetivos, expectativas, padrōes e preocupaçôes". A avaliação da QV no paciente oncológico é um importante indicador da resposta dele à doença e ao tratamento, com análise do impacto físico e psicossocial que as enfermidades, disfunçóes ou incapacidades podem causar em cada indivíduo. Portanto, a compreensão sobre a QV do paciente integra-se ao trabalho do dia a dia dos serviços de saúde, influenciando decisóes e condutas terapêuticas das equipes de saúde ${ }^{6}$.

Dessa forma, considerando a crescente incidência de câncer e, particularmente, as consequências do tratamento antineoplásico nas condições físicas e psicológicas desse público, este estudo objetivou avaliar a influência do tratamento quimioterápico no comportamento alimentar e na qualidade vida de pacientes oncológicos.

\section{METÓDO}

Trata-se de um estudo de natureza longitudinal e observacional, realizado no período de junho a outubro de 2018, no Hospital Universitário da Universidade Federal do Piauí (HU-UFPI). A amostragem foi probabilística, utilizando a técnica de amostragem aleatória simples. Partiu-se do número médio de tratamento quimioterápico do primeiro ciclo, obtido a partir de uma estimativa com base nos meses de abril a junho de 2017, na Unidade de Oncologia do HU-UFPI, resultando em uma média de dez pacientes mensais. O período de coleta seria de três meses. Dessa forma, a população total foi de 30 pacientes, considerando-se a prevalência de efeito negativo do tratamento quimioterápico no comportamento alimentar, a QV dos pacientes oncológicos de $50 \%$ e o intervalo de confiança de $90 \%$, com margem de erro de $10 \%$, atingiu-se um $n$ de 21 pacientes a serem recrutados. Contudo, em razão das perdas (óbitos, desistências e dados incompletos em prontuários), o estudo foi finalizado com 17 participantes. Os critérios de inclusão adotados foram pacientes maiores de 18 anos e de ambos os sexos, portadores de neoplasia, independentemente do tipo de tumor e tempo de diagnóstico, submetidos à quimioterapia. Foram excluídos do estudo pacientes com HIV, com problemas neurológicos ou psiquiátricos, com mais de um tipo de neoplasia, aqueles em quimioterapia paliativa e com metástase. Ressalta-se que o período de coleta de dados se estendeu por mais dois meses, em virtude da dificuldade no recrutamento, visto que nesse período ocorreram férias dos profissionais e paralisaçóes grevistas, diminuindo o fluxo de atendimentos e continuidade do tratamento quimioterápico.

A coleta de dados se deu na sala de quimioterapia, por meio de entrevista e consulta aos prontuários. Foram coletadas variáveis socioeconômicas, demográficas, clínicas, de comportamento alimentar e QV. A coleta de dados ocorreu em dois momentos: T0, antes do início do tratamento e T1, cerca de dois meses após. No primeiro momento, foram obtidos dados socioeconômicos, demográficos, clínicos, de comportamento alimentar e QV; no segundo, foram obtidas informaçóes sobre comportamento alimentar e QV.

Para descrição das características socioeconômicas e demográficas dos participantes, foram consideradas informaçôes relacionadas à data de nascimento, sexo, estado civil, cidade, etnia, grau de escolaridade, renda e hábitos de vida. Quanto aos dados clínicos, foram coletadas informaçóes sobre o tipo de câncer e tempo de diagnóstico.

A aversão alimentar foi avaliada usando a escala Food Action (FACT) utilizada no estudo de Verde ${ }^{7}$ na qual 
os alimentos foram distribuídos seguindo a estrutura da escala FACT desenvolvida por $S_{\text {chutz }}^{8}$ e agrupados de acordo com a contribuição calórica por cada $100 \mathrm{~g}$ do alimento.

Os pacientes tinham nove possibilidades de respostas, cada uma apresentava um escore determinado, variando de 1 a 9, sendo o escore de menor valor para a opção "como somente se for forçado" e o de maior valor para "como sempre que tenho oportunidade". De forma que escores maiores indicavam preferência por determinado alimento/ grupo e escores menores, aversão.

Para avaliar a aversão alimentar, as variáveis consideradas foram os alimentos e os grupos alimentares. Foram consideradas como aversóes adquiridas somente aqueles alimentos/grupos descritos a partir do tempo T1, tendo o tempo T0 como referência.

O instrumento da EORTC-QLQ-C30 versão 3.0 em português foi utilizado para a análise da $\mathrm{QV}^{9}$. Esse instrumento inclui cinco escalas funcionais (função física, função cognitiva, função emocional, função social e desempenho de papéis), três escalas de sintomas (fadiga, dor, náuseas e vômitos), uma escala de saúde global/ $\mathrm{QV}$, seis outros itens que avaliam sintomas comumente relatados por pacientes oncológicos (dispneia, falta de apetite, anorexia, insônia, constipação e diarreia), e uma escala de avaliação do impacto financeiro do tratamento e da doença.

Para a avaliação da QV, por meio do QLQ-C30, foram considerados escores para cada escala, que variavam de 0 a 100. Nas escalas funcional e medida global de saúde (MGS)/QV, quanto mais alto o escore total, mais próximo de níveis saudáveis de QV se encontrava o participante. Contudo, para as escalas de sintomas e de dificuldade financeira, quanto mais altos os valores dos escores, maiores eram os efeitos colaterais ${ }^{10}$.

As análises estatísticas foram realizadas no SPSS v.20.0. O teste de Shapiro-Wilk foi aplicado para verificar a normalidade dos dados. As variáveis quantitativas foram comparadas entre dois momentos pareados, utilizando teste $\mathrm{t}$-Student para as variáveis com distribuição normal e o teste de Wilcoxon para as variáveis não paramétricas.

As correlaçóes lineares intra e entre os tempos (T0 e T1) foram efetuadas utilizando-se o coeficiente de correlação linear de Pearson para os dados com distribuição normal, e o coeficiente de correlação de Spearman para aqueles com distribuição não paramétrica. Com significância estatística para $\mathrm{p}<0,05$, adotando-se um intervalo de confiança de $95 \%$.

A pesquisa seguiu as determinaçôes da Resolução $n^{\circ}$ 466/2012 ${ }^{11}$, sendo aprovada pelo Comitê de Ética em Pesquisa com Seres Humanos do HU/UFPI, sob parecer CAAE: 85281318.8.0000.8050.

\section{RESULTADOS}

A Tabela 1 apresenta a distribuiçáo da amostra segundo características socioeconômicas, demográficas e clínicas. Foram estudados 17 pacientes, a média de idade foi de 54,21 anos, a maioria era do sexo feminino $(82,4 \%)$, de etnia parda $(76,5 \%)$, casados $(70,6 \%)$, com ensino fundamental incompleto $(58,8 \%)$ e renda familiar entre um e dois salários mínimos $(64,7 \%)$.

Tabela 1. Distribuição da amostra estudada segundo características socioeconômicas, demográficas e clínicas

\begin{tabular}{|c|c|c|}
\hline Variáveis & $\mathbf{N}$ & $\%$ \\
\hline \multicolumn{3}{|l|}{ Sexo } \\
\hline Feminino & 14 & 82,4 \\
\hline Masculino & 3 & 17,6 \\
\hline \multicolumn{3}{|l|}{ Etnia } \\
\hline Pardo & 13 & 76,5 \\
\hline Preto & 4 & 23,5 \\
\hline \multicolumn{3}{|l|}{ Estado civil } \\
\hline Casado & 12 & 70,6 \\
\hline Solteiro & 3 & 17,6 \\
\hline Divorciado & 1 & 5,9 \\
\hline Outro & 1 & 5,9 \\
\hline \multicolumn{3}{|l|}{ Escolaridade } \\
\hline Ensino fundamental incompleto & 10 & 58,8 \\
\hline Ensino fundamental completo & 3 & 17,6 \\
\hline Ensino médio completo & 4 & 23,5 \\
\hline \multicolumn{3}{|l|}{ Renda familiar } \\
\hline Menos de 1 salário mínimo & 5 & 29,4 \\
\hline 1 a 2 salário mínimo & 11 & 64,7 \\
\hline 2 a 3 salário mínimo & 1 & 5,9 \\
\hline \multicolumn{3}{|l|}{ Tipo de câncer } \\
\hline Colo do útero & 4 & 23,5 \\
\hline Mama & 9 & 52,9 \\
\hline Ovário & 1 & 5,9 \\
\hline Próstata & 2 & 11,8 \\
\hline Reto & 1 & 5,9 \\
\hline \multicolumn{3}{|l|}{ Tempo de diagnóstico } \\
\hline$\leq 3$ meses & 9 & 53,0 \\
\hline$>3$ meses & 4 & 23,5 \\
\hline Não informado & 4 & 23,5 \\
\hline
\end{tabular}

Quanto ao perfil clínico, os cânceres mais prevalentes nessa populaçáo foram o de mama $(52,9 \%)$ e do colo do útero $(23,5 \%)$. O tempo de diagnóstico predominante foi inferior a três meses (53\%). 
A Tabela 2 apresenta os escores de aversóes alimentares segundo os alimentos nos dois momentos. Os alimentos listados a seguir são aqueles cujos resultados apresentaram significância estatística $(\mathrm{p}<0,05)$.

Tabela 2. Escores de aversões alimentares segundo os alimentos, nos momentos $\mathrm{TO}$ e $\mathrm{Tl}$

\begin{tabular}{|c|c|c|c|c|}
\hline Variáveis & Tempo & Média & $\begin{array}{l}\text { Desvio- } \\
\text {-padrão }\end{array}$ & $\mathbf{p}^{*}$ \\
\hline \multirow{2}{*}{$\begin{array}{l}\text { Macarronada, } \\
\text { lasanha }\end{array}$} & TO & 5,24 & 2,22 & \multirow{2}{*}{0,022} \\
\hline & $\mathrm{T} 1$ & 3,35 & 1,77 & \\
\hline \multirow{2}{*}{$\begin{array}{l}\text { Pizza, empada, } \\
\text { esfiha, pastel, } \\
\text { kibe, coxinha }\end{array}$} & TO & 4,53 & 2,27 & \multirow{2}{*}{0,001} \\
\hline & T1 & 1,76 & 0,97 & \\
\hline \multirow{2}{*}{$\begin{array}{l}\text { Carne de } \\
\text { charque, carne } \\
\text { de sol }\end{array}$} & TO & 4,71 & 2,39 & \multirow{2}{*}{0,028} \\
\hline & $\mathrm{T1}$ & 3,24 & 1,92 & \\
\hline \multirow{2}{*}{$\begin{array}{l}\text { Linguiça, } \\
\text { salsicha, } \\
\text { presunto, } \\
\text { outros frios }\end{array}$} & TO & 4,59 & 2,60 & \multirow[b]{2}{*}{0,004} \\
\hline & $\mathrm{T} 1$ & 2,18 & 1,88 & \\
\hline \multirow{2}{*}{$\begin{array}{l}\text { Batata, } \\
\text { mandioca, } \\
\text { inhame - } \\
\text { assado/cozido }\end{array}$} & TO & 7,71 & 1,31 & \multirow[b]{2}{*}{0,042} \\
\hline & $\mathrm{T} 1$ & 6,29 & 2,09 & \\
\hline \multirow{2}{*}{$\begin{array}{l}\text { Queijo } \\
\text { de minas } \\
\text { ou ricota, } \\
\text { requeijão light }\end{array}$} & TO & 5,35 & 2,55 & \multirow[b]{2}{*}{$0,007^{* *}$} \\
\hline & $\mathrm{T1}$ & 3,06 & 1,64 & \\
\hline \multirow{2}{*}{$\begin{array}{l}\text { Pepino, } \\
\text { pimentão }\end{array}$} & TO & 5,29 & 2,95 & \multirow{2}{*}{0,048} \\
\hline & T1 & 4,00 & 2,62 & \\
\hline \multirow{2}{*}{$\begin{array}{l}\text { Suco natural } \\
\text { de outras } \\
\text { frutas }\end{array}$} & TO & 7,41 & 2,03 & \multirow{2}{*}{0,041} \\
\hline & $\mathrm{T} 1$ & 6,41 & 1,70 & \\
\hline \multirow{2}{*}{$\begin{array}{l}\text { Pão francês, } \\
\text { forma, } \\
\text { integral, doce, } \\
\text { torrada }\end{array}$} & TO & 7,41 & 1,81 & \multirow[b]{2}{*}{0,038} \\
\hline & $\mathrm{T} 1$ & 5,65 & 2,32 & \\
\hline \multirow{2}{*}{$\begin{array}{l}\text { Manteiga } \\
\text { passada no } \\
\text { pão }\end{array}$} & TO & 4,00 & 2,45 & \multirow{2}{*}{0,003} \\
\hline & $\mathrm{T} 1$ & 1,76 & 1,79 & \\
\hline \multirow{2}{*}{$\begin{array}{l}\text { Chocolate, } \\
\text { bombons, } \\
\text { brigadeiro }\end{array}$} & TO & 3,59 & 2,12 & \multirow{2}{*}{0,038} \\
\hline & $\mathrm{T} 1$ & 2,41 & 1,50 & \\
\hline \multirow{2}{*}{ Sorvete } & TO & 4,65 & 2,15 & \multirow{2}{*}{0,004} \\
\hline & $\mathrm{T} 1$ & 2,24 & 1,79 & \\
\hline
\end{tabular}

Legendas: *Teste de Wilcoxon; ${ }^{* *}$ Teste t-Student.

Para todos os alimentos, os valores do escores foram menores após o tratamento quimioterápico (T1), com destaque para os alimentos "pizza, empada, esfiha, pastel, kibe, coxinha” ( $p=0,001)$, "linguiça, salsicha, presunto, outros frios" ( $\mathrm{p}=0,004)$; "manteiga passada no pão" $(p=0,003)$; "sorvete" $(p=0,004)$; "queijo de minas ou ricota, requeijão light" $(\mathrm{p}=0,007)$.

Ao se analisar os escores de aversão para grupos de alimentos (Tabela 3), verificou-se uma redução significativa nos escores do grupo de "sopas e massas" $(\mathrm{p}=0,001)$, "carnes e peixes" $(\mathrm{p}=0,016)$ e "doces, sobremesas e aperitivos" ( $\mathrm{p}=0,001)$, entre os tempos T0 e T1. Os demais grupos de alimentos apresentaram tendência à redução nos valores médios de escores obtidos, em T1, contudo, sem significância estatística.

Tabela 3. Escores de aversões alimentares segundo os grupos de alimentos, nos momentos $\mathrm{TO}$ e $\mathrm{Tl}$

\begin{tabular}{l|c|c|c|c}
\hline \multicolumn{1}{c|}{ Variáveis } & Tempo & Média & $\begin{array}{c}\text { Desvio- } \\
\text {-padrão }\end{array}$ & $\mathbf{p}^{*}$ \\
\hline Sopas e & T0 & 22,71 & 4,73 & 0,001 \\
massas & T1 & 16,41 & 4,37 & \\
Carnes e & T0 & 54,41 & 11,53 & 0,016 \\
peixes & T1 & 43,71 & 9,15 & \\
Leguminosas & T0 & 21,35 & 4,43 & 0,195 \\
e ovo & T1 & 19,53 & 4,02 & \\
Arroz e & T0 & 32,71 & 6,34 & 0,244 \\
tubérculos & T1 & 29,94 & 7,22 & \\
Leite/derivados & T0 & 35,41 & 8,08 & 0,067 \\
e cereais & T1 & 30,82 & 5,88 & \\
Vegetais & T0 & 35,12 & 10,42 & 0,159 \\
& T1 & 32,00 & 7,57 & \\
Molhos & T0 & 12,53 & 6,17 & 0,256 \\
& T1 & 9,88 & 5,22 & \\
Frutas e sucos & T0 & 62,24 & 14,54 & 0,065 \\
& T1 & 55,94 & 8,47 & \\
Pães e & T0 & 17,76 & 4,19 & 0,056 \\
biscoitos & T1 & 14,47 & 5,30 & \\
Bebidas & T0 & 14,41 & 6,71 & $0,126 * *$ \\
Doces, & T1 & 11,76 & 4,29 & \\
sobremesas e & T0 & 29,59 & 5,46 & 0,001 \\
aperitivos & T1 & 21,29 & 7,87 & \\
escores & T0 & 338,29 & 47,72 & 0,002 \\
\hline & T1 & 282,82 & 40,09 & \\
\hline
\end{tabular}

Legendas: *Teste t-Student; ${ }^{* *}$ Teste Wilcoxon.

Em relação à $\mathrm{QV}$, a Tabela 4 mostra as variáveis analisadas com os escores correspondentes aos dois momentos do tratamento quimioterápico. Observa-se alteração na percepção da $\mathrm{QV}$ pelos pacientes submetidos ao estudo, quando se comparou T0 e T1, para os domínios: MGS ( $\mathrm{p}=0,001)$ e "dificuldade financeira" 
$(\mathrm{p}=0,026)$. Para as demais variáveis, não houve alteraçôes significativas entre os tempos T0 e T1.

Tabela 4. Escores segundo escalas e domínios do EORTC QLQ-C30 nos momentos TO e T1

\begin{tabular}{|c|c|c|c|c|}
\hline Variáveis & Tempo & Média & $\begin{array}{l}\text { Desvio- } \\
\text {-padrão }\end{array}$ & p* \\
\hline \multirow{2}{*}{$\begin{array}{l}\text { Medida global } \\
\text { de saúde }\end{array}$} & TO & 66,18 & 25,25 & \multirow{2}{*}{0,001} \\
\hline & $\mathrm{T} 1$ & 86,27 & 18,85 & \\
\hline \multirow{2}{*}{$\begin{array}{l}\text { Escala } \\
\text { funcional }\end{array}$} & TO & 21,82 & 14,92 & \multirow{2}{*}{0,836} \\
\hline & $\mathrm{T} 1$ & 20,27 & 18,99 & \\
\hline \multirow{2}{*}{ Função física } & TO & 22,35 & 22,23 & \multirow{2}{*}{0,479} \\
\hline & $\mathrm{T1}$ & 25,88 & 22,47 & \\
\hline \multirow{2}{*}{$\begin{array}{l}\text { Desempenho } \\
\text { de papéis }\end{array}$} & TO & 17,65 & 27,30 & \multirow{2}{*}{0,385} \\
\hline & $\mathrm{T} 1$ & 21,57 & 25,85 & \\
\hline \multirow{2}{*}{$\begin{array}{l}\text { Função } \\
\text { emocional }\end{array}$} & TO & 28,92 & 25,02 & \multirow{2}{*}{0,074} \\
\hline & $\mathrm{T} 1$ & 16,67 & 25,85 & \\
\hline \multirow{2}{*}{$\begin{array}{l}\text { Função } \\
\text { cognitiva }\end{array}$} & TO & 16,67 & 23,57 & \multirow{2}{*}{0,593} \\
\hline & $\mathrm{T1}$ & 14,71 & 26,27 & \\
\hline \multirow{2}{*}{ Função social } & TO & 23,53 & 28,30 & \multirow{2}{*}{0,877} \\
\hline & $\mathrm{T} 1$ & 22,55 & 30,01 & \\
\hline \multirow{2}{*}{$\begin{array}{l}\text { Escala de } \\
\text { sintomas }\end{array}$} & TO & 21,08 & 13,19 & \multirow{2}{*}{$0,851^{*}$} \\
\hline & $\mathrm{T} 1$ & 20,30 & 16,57 & \\
\hline \multirow{2}{*}{ Fadiga } & TO & 29,41 & 30,67 & \multirow{2}{*}{0,488} \\
\hline & $\mathrm{T1}$ & 33,99 & 29,26 & \\
\hline \multirow{2}{*}{$\begin{array}{l}\text { Náusea e } \\
\text { vômito }\end{array}$} & TO & 9,80 & 17,74 & \multirow{2}{*}{0,259} \\
\hline & $\mathrm{T1}$ & 18,63 & 23,48 & \\
\hline \multirow{2}{*}{ Dor } & TO & 31,37 & 27,56 & \multirow{2}{*}{0,263} \\
\hline & $\mathrm{T1}$ & 21,57 & 28,11 & \\
\hline \multirow{2}{*}{ Dispneia } & TO & 11,76 & 23,40 & \multirow{2}{*}{0,334} \\
\hline & $\mathrm{T} 1$ & 5,88 & 13,10 & \\
\hline \multirow{2}{*}{ Insônia } & TO & 33,33 & 40,82 & \multirow{2}{*}{0,167} \\
\hline & $\mathrm{T} 1$ & 17,65 & 26,66 & \\
\hline \multirow{2}{*}{$\begin{array}{l}\text { Perda de } \\
\text { apetite }\end{array}$} & TO & 25,49 & 36,38 & \multirow{2}{*}{0,915} \\
\hline & $\mathrm{T1}$ & 25,49 & 38,24 & \\
\hline \multirow{2}{*}{ Constipação } & TO & 19,61 & 29,01 & 36 \\
\hline & $\mathrm{T1}$ & 15,69 & 29,15 & 0,300 \\
\hline & TO & 7,84 & 25,08 & \\
\hline Diarreia & $\mathrm{T} 1$ & 23,53 & 30,65 & 0,00 \\
\hline Dificuldade & TO & 50,98 & 44,28 & 0,026 \\
\hline financeira & $\mathrm{T} 1$ & 23,53 & 34,89 & $0,0<0$ \\
\hline
\end{tabular}

Legendas: *Teste Wilcoxon; ${ }^{* *}$ Teste t-Student.

Foram analisadas também as possíveis correlaçôes entre a QV e o comportamento alimentar nos dois momentos, cujos resultados são descritos na Tabela 5. De acordo com esses resultados, observaram-se correlaçôes significativas entre essas variáveis no momento $\mathrm{T} 1$, as quais não existiam antes do tratamento quimioterápico (T0).

Observa-se que, em relação à MGS, "frutas e sucos"; e "doces, sobremesas e aperitivos" apresentaram uma correlação positiva e estatisticamente significativa $(\mathrm{p}=0,040$ e $\mathrm{p}=0,031)$ no momento T0. Referente à escala funcional, o estudo apontou uma correlação negativa e significativa para o grupo de "doces, sobremesas e aperitivos" ( $\mathrm{p}=0,003)$ e "carnes e peixes" ( $\mathrm{p}=0,020)$. Em relação à escala de sintomas, também houve correlação negativa significativa com o seu aparecimento e o consumo de "leguminosas, ovos" ( $\mathrm{p}=0,047)$, "molhos" ( $\mathrm{p}=0,024)$ e "bebidas" $(0,028)$.

Tabela 5. Correlação entre QV e comportamento alimentar nos momentos TO e T1

\begin{tabular}{|c|c|c|c|}
\hline $\begin{array}{l}\text { Escalas } \\
\text { do EORTC } \\
\text { QLQ-C30 }\end{array}$ & $\begin{array}{l}\text { Variável de } \\
\text { correlação }\end{array}$ & $\mathbf{r}$ & p* \\
\hline \multicolumn{4}{|c|}{ TO } \\
\hline \multirow[b]{2}{*}{$\begin{array}{l}\text { Medida global } \\
\text { de saúde }\end{array}$} & Frutas e sucos & 0,503 & 0,040 \\
\hline & $\begin{array}{l}\text { Doces, } \\
\text { sobremesas e } \\
\text { aperitivos }\end{array}$ & 0,524 & 0,031 \\
\hline \multirow[b]{2}{*}{$\begin{array}{l}\text { Escala } \\
\text { funcional }\end{array}$} & Frutas e sucos & $-0,632$ & 0,006 \\
\hline & $\begin{array}{l}\text { Doces, } \\
\text { sobremesas e } \\
\text { aperitivos }\end{array}$ & $-0,676$ & 0,003 \\
\hline \multirow{4}{*}{$\begin{array}{l}\text { Escala de } \\
\text { sintomas }\end{array}$} & Carnes e peixes & $-0,58$ & 0,020 \\
\hline & $\begin{array}{l}\text { Leguminosas e } \\
\text { ovo }\end{array}$ & $-0,488$ & 0,047 \\
\hline & Molhos & $-0,543$ & 0,024 \\
\hline & Bebidas & $-0,531$ & 0,028 \\
\hline \multirow{4}{*}{$\begin{array}{l}\text { Dificuldade } \\
\text { financeira }\end{array}$} & $\begin{array}{l}\text { Leite/derivados } \\
\text { e cereais }\end{array}$ & $-0,486$ & $0,048^{* *}$ \\
\hline & Vegetais & $-0,593$ & $0,012^{* *}$ \\
\hline & Frutas e sucos & $-0,602$ & $0,011^{* *}$ \\
\hline & $\begin{array}{l}\text { Doces, } \\
\text { sobremesas e } \\
\text { aperitivos }\end{array}$ & $-0,555$ & $0,021^{* *}$ \\
\hline \multicolumn{4}{|c|}{ T1 } \\
\hline $\begin{array}{l}\text { Dificuldade } \\
\text { financeira }\end{array}$ & Carnes e peixes & 0,486 & $0,048^{* *}$ \\
\hline
\end{tabular}

Legendas: *Correlaçăo de Pearson; **Correlaçấo de Spearman.

\section{DISCUSSÃO}

Considerando a crescente incidência de câncer e, particularmente, as consequências do tratamento antineoplásico nas condiçôes físicas e psicológicas desse público, reforça-se a necessidade de estudos que 
investiguem a influência, em médio e longo prazos, do tratamento quimioterápico no comportamento alimentar e na qualidade vida desses pacientes.

Quanto ao sexo, o resultado do presente trabalho foi semelhante ao estudo de Hisse et al. ${ }^{12}$, o qual objetivou conhecer as características sociodemográficas e econômicas, as morbidades autorreferidas e os hábitos de vida das pessoas com câncer em tratamento quimioterápico e hormonioterápico atendidas na unidade de oncologia de um hospital de ensino na Regiáo Sul do Brasil. Nesse estudo, 52,9\% de participantes eram do sexo feminino. Em relação à etnia, houve divergência, visto que no presente estudo a maioria $(76,5 \%)$ era parda. Enquanto no de Hisse et al. ${ }^{12}$, houve predomínio $(81,5 \%)$ de indivíduos de cor branca, o que sugere ter sido por causa das diferenças existentes entre as regiôes do Brasil onde foram realizados, tendo a Regiấo Sul maior prevalência de indivíduos caucasianos e de colonização europeia.

Em relaçáo ao tipo de câncer, os de maior prevalência foram: de mama $(52,9 \%)$, do colo do útero $(23,5 \%)$ e de próstata $(11,8 \%)$. Esses resultados seguem as tendências apontadas por Ferlay et al. ${ }^{13}$ e corroboram os achados de outros estudos ${ }^{14,15}$ realizados com pacientes oncológicos sem tratamento quimioterápico. Ressalta-se, ainda, que a maior frequência de neoplasias da mama e do colo do útero pode ser em razão predominância do sexo feminino na população estudada.

O tempo de diagnóstico predominante foi o inferior a três meses (53\%), dado que corrobora achados de Souza et al. ${ }^{16}$, os quais obtiveram tempo mediano de 45 dias, em estudo com mulheres maiores de 60 anos diagnosticadas com câncer de mama em São Paulo; e o de Paiva et al. ${ }^{17}$, cujo tempo de diagnóstico foi inferior a 30 dias em $88,9 \%$ dos casos. Destaca-se que a precocidade no diagnóstico das neoplasias repercute diretamente na sobrevida dos pacientes.

As aversões alimentares adquiridas em pacientes com câncer resultam da associaçáo entre sensação de mal-estar, que ocorre em consequência do tratamento antineoplásico, e o sabor do alimento simultaneamente consumido. $\mathrm{O}$ alimento é entáo interpretado como ruim e, consequentemente, evitado, o que traz implicaçóes nutricionais, afetando o estado nutricional e a QV dos pacientes ${ }^{18}$.

Em relação à avaliação das aversões alimentares nos dois momentos propostos, constatou-se redução dos escores de preferências alimentares após o tratamento quimioterápico (T1) para todos os alimentos, com destaque para "pizza, empada, esfiha, pastel, kibe, coxinha" ( $\mathrm{p}=0,001)$; "linguiça, salsicha, presunto, outros frios" ( $\mathrm{p}=0,004)$; "manteiga passada no pão" $(\mathrm{p}=0,003)$; "sorvete" $(\mathrm{p}=0,004)$ e "suco natural de outras frutas" ( $\mathrm{p}=0,041)$; "queijo de minas ou ricota, requeijão light” $(\mathrm{p}=0,007)$, indicando aversão após o tratamento quimioterápico. Diferentemente dos resultados obtidos nos estudos de Verde ${ }^{7}$, em trabalho realizado com 25 pacientes portadoras de neoplasia mamária, foi verificada uma redução apenas para o café preto, após o início do tratamento quimioterápico e o de Silva et al. ${ }^{1}$, no qual náo foi observada diferença significativa nas aversóes alimentares relatadas pelos pacientes entre os momentos T0 e T1.

A reduçáo no consumo de sucos naturais e de frutas pode ser prejudicial para esses pacientes, visto que acarreta diminuição na ingestấo de nutrientes antioxidantes, que são primordiais para proteção do organismo contra a ação de radicais livres ${ }^{19}$. Dessa forma, ressalta-se a importância de incentivar esses pacientes a consumirem frutas e vegetais, visto que são fontes naturais de nutrientes antioxidantes, os quais conferem efeito protetor, estando associados a uma melhor sobrevida.

Marinho et al. ${ }^{20}$, analisando a populaçáo em três períodos (antes, durante e depois da quimioterapia), verificaram, em pacientes com câncer de mama, apetite maior por alimentos salgados nos tempos $\mathrm{T} 1 \mathrm{e} \mathrm{T} 2$ do que em T0, enquanto os alimentos condimentados foram mais bem avaliados em T1; resultados similares aos obtidos no presente estudo.

Com relação ao consumo de carne vermelha, verificou-se uma diminuição significativa $(\mathrm{p}=0,016)$ nos escores de preferência para o grupo das carnes e peixes. Resultado divergente do obtido no estudo de Verde ${ }^{7}$, no qual foi constatado aumento significativo no escore de preferências para carne bovina (cozida, assada, grelhada ou churrasco). Capelari et al. ${ }^{21}$, em estudo com 100 pacientes em tratamento quimioterápico, observaram predominância de aversóes alimentares relacionadas à carne, comidas gordurosas, doces e sólidos em geral, resultado similar aos obtidos no presente estudo.

Em relação aos produtos lácteos, também foi observada uma redução nos escores de preferências em $\mathrm{T} 1$, resultado divergente do obtido por Verde ${ }^{7}$, que identificou, em pacientes com câncer de mama, a presença de aversão alimentar pós-quimioterapia para produtos em $23 \%$ das pacientes e citaram leite e derivados como sendo associado a incômodo no T1.

Possivelmente, a redução nos escores de preferências por alguns alimentos possa ter sido influenciada pelas orientaçôes alimentares que recebem ao iniciarem a tratamento quimioterápico ou, até mesmo, pelos efeitos colaterais provocados pelo próprio tratamento, que têm repercussão na ingestão alimentar, além de outros fatores como: o tipo de protocolo quimioterápico, o uso de antieméticos, entre outros fatores que não foram considerados neste estudo. 
No que se refere à $\mathrm{QV}$, constatou-se que os pacientes estudados consideram como satisfatória sua QV em T0, permanecendo assim em T1. Diferentemente do verificado no estudo de Miranda et al. ${ }^{22}$, com 60 pacientes oncológicos de um hospital universitário de Belém, que utilizou a mesma ferramenta de avaliação para a QV, com média de 11,4, demonstrando que os pacientes consideravam sua QV como insatisfatória.

Os estudos de Fachinello et al. ${ }^{23}$ e Vendrusculo ${ }^{9}$ obtiveram resultados semelhantes aos obtidos no presente estudo, o primeiro realizado com dez pacientes em início do tratamento oncológico demonstrou MSG/QV de T0 (32) e T1 $(72,1)$, com diferença estatística; o segundo, com pacientes diagnosticadas com câncer de mama, após início do tratamento, MSG/QV de 70,1. Possivelmente, isso ocorre em virtude da melhora no estado de saúde dos pacientes e da diminuição dos sintomas após os primeiros meses de tratamento ${ }^{23}$, ou ainda, da atuação da equipe multiprofissional, promovendo açóes de acolhimento e acompanhamento desde o início do tratamento.

Outro domínio da escala de QV que foi significativo entre T0 e T1 foi "dificuldade financeira", com diminuição dos escores desse domínio em T1, demonstrando melhora da QV. Achado que corrobora os de Sawada et al. ${ }^{24}$ com 30 pacientes neoplásicos apontou que, em relação à dificuldade financeira, o escore médio encontrado foi de 35,5 , demonstrando que, apesar de o tratamento decorrente da doença ser complexo e oneroso, esse fato não trouxe dificuldades financeiras significativamente relevantes que interferissem na $\mathrm{QV}$ desses pacientes.

A escolha alimentar é o resultado de uma interação complexa entre fatores intra e interpessoais. Os determinantes das escolhas alimentares podem ser classificados como biológicos (fome, paladar e apetite), econômicos (custo, renda e disponibilidade), físicos (acesso, educação, instalaçóes de cozinha e tempo), sociais (cultura, família, pares e padrōes de refeições) e psicológicos (disposição, estresse e culpa), além de atitudes, crenças e conhecimentos sobre alimentação. $\mathrm{Na}$ população em geral, a escolha de alimentos é baseada no sabor, custo, conveniência, saúde e variedade, e o sabor é percebido como um fator altamente importante nas decisôes de escolha de alimentos ${ }^{25}$.

Nesse sentido, foi analisada a correlação entre QV e comportamento alimentar, sendo identificada correlaçáo positiva e significativa em $\mathrm{T} 1$ apenas para o domínio "dificuldade financeira" e consumo de carnes e peixes, mostrando que esses alimentos foram priorizados na segunda fase do estudo, o que pode apontar um maior nível de informaçáo obtida no que diz respeito ao aumento do consumo de proteínas como mudança alimentar durante o tratamento quimioterápico.
O estudo de Campos et al. ${ }^{26}$ mostrou que a ingestão dietética não estava relacionada de forma significativa com a QV de pacientes com câncer. Por outro lado, destaca-se a influência significativa do apetite e dos sintomas da doença para a QV desses pacientes, bem como de variáveis clínicas e demográficas, corroborando outros estudos ${ }^{27,28}$. Esses achados reforçam a necessidade de monitoramento nutricional desde o momento do diagnóstico, no intuito de propiciar melhorias na QV desses pacientes.

Ressalta-se que este estudo apresentou algumas limitações, como: tamanho amostral, instrumento de avaliação das aversôes alimentares, intervalo entre os dois momentos estudados, e fatores de confundimento não considerados nas análises.

\section{CONCLUSÃO}

Os achados deste estudo mostraram que a população era composta, em sua maioria, de mulheres pardas com diagnóstico de câncer de mama. Concluiu, ainda, que houve diferença significativa entre os tempos $\mathrm{T} 0$ e $\mathrm{T} 1$ nos escores de preferências alimentares de diversos alimentos de forma isolada e de alguns grupos.

Os pacientes apontaram como satisfatória a sua QV. O domínio "dificuldade financeira" apresentou correlação significativa com preferência por alguns alimentos em T1. Os resultados obtidos reforçam a necessidade de constante monitoramento nutricional, desde o diagnóstico e durante o tratamento quimioterápico, no intuito de minimizar e/ ou evitar as repercussōes negativas ocasionadas por essa doença no estado nutricional e, consequentemente, na QV desses indivíduos. Além disso, sugere-se a realização de mais estudos, considerando as limitaçóes apresentadas.

\section{CONTRIBUIÇÕES}

Ana Letícia Pereira Andrade, Emilene Maciel e Maciel, Suelem Torres de Freitas e Maria da Cruz Moura e Silva participaram da concepção e planejamento do estudo, na obtenção, análise e/ou interpretação dos dados, assim como na redação e/ou revisão crítica e aprovação final da versão publicada; Gilmara Péres Rodrigues participou da redação e/ou revisão crítica e aprovação final da versão publicada.

\section{DECLARAÇÃO DE CONFLITO DE INTERESSE}

Nada a declarar.

\section{FONTES DE FINANCIAMENTO}

Não há. 


\section{REFERÊNCIAS}

1. Silva AM, Franco LP, Santos TSS, et al. Impacto das aversóes alimentares no estado nutricional de pacientes oncológicos submetidos à quimioterapia. J Health Sci Inst. 2012;30(2):166-70.

2. Coa KI, Epstein JB, Ettinger D, et al. The impact of cancer treatment on the diets and food preferences of patients receiving outpatient treatment. Nutr Cancer. 2015;67(2),339-353. doi: https://doi.org/10.1080/016 35581.2015.990577

3. Silvano LVM, Oliveira CLA. Perfil nutricional de pacientes oncológicos Internados em um hospital militar do município de Natal - RN. Revista UNI-RN. 2014;13(1/2):125-143.

4. Palazzo CC. Alimentação, sensibilidade e preferência ao gosto doce na quimioterapia para o câncer de mama [dissertação]. Ribeirão Preto (SP): Faculdade de Medicina de Ribeirão Preto; 2016. doi: https://doi. org/10.11606/D.17.2016.tde-29082016-101248

5. World Health Organization. Obesity: preventing and managing the global epidemic: report of a WHO Consultation. Geneva: WHO; 2000. (WHO technical report series; 894). Part I, The problem of overweight and obesity; p. 5.

6. Nicolussi AC, Sawada NO, Cardozo FMC, et al. Qualidade de vida relacionada à saúde de pacientes com câncer em quimioterapia. Rev Rene. 2014 jan.-fev.; 15(1):132-40. doi: https://doi.org/10.15253/21756783.2014000100017

7. Verde SMML. Impacto do tratamento quimioterápico no estado nutricional e no comportamento alimentar de pacientes com neoplasia mamária e suas conseqüências na qualidade de vida [dissertação]. São Paulo: Universidade de São Paulo; 2007.

8. Schutz HG. Food action rating scale for measuring food acceptance. J Food Sci. 1965;30(2):365-74. doi: https:// doi.org/10.1111/j.1365-2621.1965.tb00316.x

9. Vendrusculo LM. Capacidade funcional e qualidade de vida de mulheres com câncer de mama após o tratamento oncológico [dissertação]. Ribeirão Preto (SP): Escola de Enfermagem de Ribeirão Preto, Universidade de São Paulo; 2011. doi: https://doi.org/10.11606/D.22.2011. tde-15082012-135511

10. Fayers PM, Aaronson NK, Bjordal K, et al. EORTC QLQ-C30 scoring manual. 3th ed. Brussels: EORTC; 2001.

11. Conselho Nacional de Saúde (BR). Resolução no 466, de 12 de dezembro de 2012. Diário Oficial da União, Brasília, DF; 2013 jun. 13. Seção I, p. 59.

12. Hisse CN, Schwartz E, Lima LM, et al. Caracterização dos pacientes de quimioterapia e hormonioterapia de uma unidade de oncologia. Rev Enferm Cent-Oeste Min. 2014 maio-ago; 4(2):1185-1193. doi: http://dx.doi. org/10.19175/recom.v0i0.492
13. Ferlay J, Colombet M, Soerjomataram I, et al. Estimating the global cancer incidence and mortality in 2018: GLOBOCAN sources and methods. Int J Cancer. 2019;144(8):1941-1953. doi: https://doi.org/10.1002/ ijc.31937

14. Borges LR. Fatores determinantes da qualidade de vida em uma coorte de pacientes submetidos à quimioterapia [dissertação]. Pelotas (RS): Universidade Católica de Pelotas; 2008.

15. Nicolussi AC, Cardozo FMC, Paula JM, et al. Relaxamento com imagem guiada e qualidade de vida relacionada à saúde de pacientes durante quimioterapia. Rev Enferm Atenção Saúde. 2018;7(2):17-31. doi: https://doi.org/10.18554/reas.v7i2.2130

16. Souza CB, Fustinoni SM, Amorim MHC, et al. Estudo do tempo entre o diagnóstico e início do tratamento do câncer de mama em idosas de um hospital de referência em São Paulo, Brasil. Ciênc Saúde Coletiva. 2015;20(12):3805-3816. doi: http://dx.doi. org/10.1590/1413-812320152012.00422015

17. Paiva CJK, Cesse EAP. Aspectos relacionados ao atraso no diagnóstico e tratamento do câncer de mama em uma unidade hospitalar de Pernambuco. Rev Bras Cancerol. 2015;61(1): 23-30. doi: https://doi.org/10.32635/21769745.RBC.2015v61n 1.374

18. Fisberg RM, Slater B, Marchioni DML, et al. Inquéritos alimentares: métodos e bases científicos. Barueri, SP: Manole; 2005.

19. Rohenkohl CC, Carniel AP, Colpo E. Consumo de antioxidantes durante tratamento quimioterápico. ABCD, Arq Bras Cir Dig. 2011;24(2):107-112. doi: http://dx.doi.org/10.1590/S0102-67202011000200004

20. Marinho EDC, Custódio IDD, Ferreira IB, et al. Impact of chemotherapy on perceptions related to food intake in women with breast cancer: a prospectiv estudy. PLoS One. 2017;12(11):e0187573. doi: https://doi. org/10.1371/journal.pone.0187573

21. Capelari P, Ceni GC. Feeding behavior and nutritional profile of cancer patients in drug therapy. Demetra. 2018;13(1):223-240. doi: https://doi.org/10.12957/ demetra.2018.30309

22. Miranda TV, Neves FMG, Costa GNR, et al. Estado nutricional e qualidade de vida de pacientes em tratamento quimioterápico. Rev Bras Cancerol. 2013;59(1):57-64.

23. Fachinello T, Confortin FG. Avaliação do estado nutricional, qualidade de vida e aversôes alimentares de pacientes em tratamento oncológico em um hospital do oeste de Santa Catarina [trabalho de conclusão]. Unochapecó (SC): Curso de Pós-Graduação Lato Sensu em Nutrição Humana, Universidade Comunitária da Região de Chapecó; 2013.

24. Sawada NO, Machado SM. Avaliação da qualidade de vida de pacientes oncológicos em tratamento 
quimioterápico adjuvante. Texto Contexto Enferm. 2008;17(4):750-7. doi: http://dx.doi.org/10.1590/ S0104-07072008000400017

25. Castro MC, Araújo SA, Mendes TR, et al. Effectiveness of antiemetics in control of antineoplastic chemotherapyinduced emesis at home. Acta Paul Enferm. 2014;27(5):412-8. doi: http://dx.doi.org/10.1590/19820194201400069

26. Campos JADB, Silva WR, Spexoto MCB, et al. Características clínicas, dietéticas e demográficas que interferem na qualidade de vida de pacientes com câncer. Einstein (São Paulo). 2018;16(4): eAO4368. doi: http:// dx.doi.org/10.31744/einstein_journal/2018AO4368

27. Laird BJ, Fallon M, Hjermstad MJ, et al. Quality of life in patients with advanced cancer: differential association with performance status and systemic inflammatory response. J Clin Oncol. 2016;34(23):2769-75. doi: http://dx.doi.org/10.1200/JCO.2015.65.7742

28. Barajas Galindo DE, Vidal-Casariego A, CallejaFernández A, et al. Appetite disorders in cancer patients: impact on nutritional status and quality of life. Appetite. 2017;114:23-7. doi: http://dx.doi.org/10.1016/j. appet.2017.03.020 